\section{SAT0254 PATIENT CHARACTERISTICS AND DISEASE MANAGEMENT OF RHEUMATOID ARTHRITIS AT BASELINE IN THE VIGOUR (VIOXX GI OUTCOMES RESEARCH) STUDY: EFFECTS OF DISEASE DURATION}

${ }^{1} \mathrm{C}$ Bombardier, ${ }^{2} \mathrm{M}$ Hochberg, ${ }^{3} \mathrm{~L}$ Laine, ${ }^{4} \mathrm{DR}$ Ramey, ${ }^{4} \mathrm{~A}$ Reicin, ${ }^{4} \mathrm{DJ}$ Watson. ${ }^{1} \mathrm{Cl}$ linical Epidemiology, University of Toronto, Toronto, Canada; ${ }^{2}$ University of Maryland, Baltimore, MD, USA; ${ }^{3}$ University Southern California, Los Angeles, CA, USA; ${ }^{4}$ Merck Research Labs, West Point, PA, USA

\subsection{6/annrheumdis-2001.890}

Background The VIGOUR Study was a large $(n=8076)$ randomised, double-blind, clinical trial comparing the GI safety of rofecoxib $50 \mathrm{mg}$. once daily to naproxen $500 \mathrm{mg}$ twice daily. The median duration of follow-up was 9.0 months.

Objectives

Methods We examined demographics, disease status, disease impact, history of GI problems, and medication history for 3 subgroups based on disease duration: $0-2$ years $(\mathrm{N}=1498)$, $>2-10$ years $(\mathrm{N}=3382)$, and $>10$ years $(\mathrm{N}=3196)$.

Results Baseline demographic characteristics were quite comparable for the 3 groups. $76 \%, 78 \%$, and $83 \%$ of the patients were female, with mean ages of 57,58 , and 59 years for $0-2,>2-10$, and $>10$ years, respectively. The majority of patients were in ARA functional class II (0-2 years: 57\%, 2-10 years: 55\%, >10 years: $51 \%$ ). $67 \%$ of the $0-2$ year group were known to be seropositive compared with $74 \%$ and $75 \%$ in the other groups. The proportion of patients with erosions on hand x-rays varied by duration $(44 \%, 60 \%, 71 \%)$, as did the percentage with rheumatoid nodules $(16 \%, 30 \%, 43 \%)$.

The mean modified Health Assessment Questionnaire (mHAQ) scores (U. S. patients only) were $0.62,0.57$, and 0.61 on a scale of $0-3$ with mean patient and investigator global scores of $2.0,1.9,2.0$ and $1.9,1.8,1.9$ on scales of $0-4.56 \%$, $50 \%$, and $50 \%$ of the patients reported that they cut down their activities due to their RA for an average of 11, 10, and 10 days in the past 4 weeks. Patients reported that pain interfered with their normal work to a moderate extent $(2.9,2.8$, and 2.9 on a scale from 1 (not at all) to 5 (extremely)).

The proportions of patients taking NSAIDs at baseline were similar $(81 \%, 82 \%, 84 \%)$ as were the proportions taking corticosteroids $(57 \%, 55 \%, 56 \%)$. At baseline, patients had taken an average of 2.0, 2.8, and 3.6 DMARDs with the following proportions having taken individual medications at some time: MTX: 57\%, 74\%, 77\%; corticosteroids: 75\%, 82\%, 85\%; hydroxychloroquine: 41\%, 55\%, 59\%, injectable gold: $6 \%$, $20 \%$, 49\%, sulfasalazine: $15 \%, 26 \%, 29 \%$. $6 \%, 8 \%$, and $9 \%$ of the patients reported a prior history of a GI ulcer or bleed at baseline, and 21\%, 21\%, and 20\% reported dyspepsia within 14 days prior to baseline. 9\%, 11\%, and 14\% had stopped an arthritis medication at some time due to abdominal pain. $45 \%$ of the 0-2 year group had changed their arthritis medication in the previous year; compared to $39 \%$ in the $2-10$ year group, and $37 \%$ in the $>10$ year group.

Conclusion The VIGOUR cohort is useful for studying the relationship between disease duration and patient characteristics and treatment strategies. Despite having RA for $<2$ years, the early RA group demonstrates moderate levels of functional impairment comparable to the other groups as indicated by mHAQ and patient and investigator global scores. Patients in the early RA group also reported the greatest need to cut down their activities. The data on medication use reflect the general trends toward early use of single DMARDs and combination therapy, as well as a continuing reliance on corticosteroids. All groups were comparable in their history of serious GI events and GI side effects.

\section{SAT0255 REGIONAL VARIATION IN BASELINE PATIENT CHARACTERISTICS AND DISEASE MANAGEMENT OF RHEUMATOID ARTHRITIS PATIENTS IN THE VIGOUR (VIOXX GI OUTCOMES RESEARCH) STUDY}

${ }^{1} \mathrm{C}$ Bombardier, ${ }^{2} \mathrm{M}$ Hochberg, ${ }^{3} \mathrm{~L}$ Laine, ${ }^{4}$ DR Ramey, ${ }^{4} \mathrm{~A}$ Reicin, ${ }^{4} \mathrm{DJ}$ Watson. ${ }^{1}$ Clinical Epidemiology, University of Toronto, Toronto, Canada; 'University of Maryland, Baltimore, MD, USA; ${ }^{3}$ University of Southern California, Los Angeles, CA, USA; ${ }^{4}$ Merck Research Labs, West Point, PA, USA

\subsection{6/annrheumdis-2001.891}

Background The VIGOUR Study was a large $(\mathrm{n}=8076)$ randomised, double-blind, clinical trial comparing the GI safety of rofecoxib $50 \mathrm{mg}$. once daily to naproxen $500 \mathrm{mg}$ twice daily. The median duration of follow-up was 9.0 months.

Objectives To describe the baseline patient characteristics and prior drug treatment by geographic region, for one of the largest cohorts of RA patients ever studied.

Methods We examined demographic characteristics, disease status, history of GI problems, and medication history for subgroups of patients from Europe (EUR, $\mathrm{N}=1051$ ); Latin America (LA, $\mathrm{N}=2746$ ); and North America (NA, $\mathrm{N}=3795$ ). Results Baseline demographic characteristics were fairly comparable across regions. Most patients were female: $79 \%$ in EUR, $88 \%$ in LA, and $74 \%$ in NA. The respective mean ages were 58 , 56 , and 60 years, with 23\%, 18\%, and $32 \%$ over age 65 . The participants had mean disease duration of 11,10 , and 11 years, and the majority of patients were in ARA functional class II (54\%, 56\%, 53\%). In EUR, 70\% were known to be seropositive with $81 \%$ in LA and $68 \%$ in NA. The proportions of patients known to have erosions on hand $\mathrm{x}$-rays varied across regions $(86 \%, 73 \%, 45 \%)$, as did the percentage with rheumatoid nodules $(25 \%, 23 \%, 43 \%)$. The mean patient global disease activity scores were $2.2,2.0$, and 1.9 on a scale of $0-4$, and mean investigator global disease activity scores were $2.1,1.8$, and 1.9 on a scale of $0-4$. While the proportions of patients taking NSAIDs at baseline were similar $(87 \%, 84 \%, 81 \%)$, the proportion taking corticosteroids in LA (72\%) was much higher than in EUR (52\%) and NA (46\%). At baseline, patients had taken an average of $3.4,2.8$, and 3.0 DMARDs with the following proportions having ever taken individual medications in EUR, LA, and NA respectively: MTX: 70\%, 76\%, 71\%; corticosteroids: $81 \%$, 88\%, 79\%; hydroxychloroquine: 43\%, 62\%, 51\%, injectable gold: 49\%, 21\%, 29\%, sulfasalazine: 56\%, 15\%, 21\%. The newest DMARDs had been used by only a handful of patients in EUR and LA; in NA, 4\% had used etanercept and 7\% had used leflunomide. $7 \%, 7 \%$, and $9 \%$ of the patients reported a prior history of a GI ulcer or bleed at baseline, and 17\%, 24\%, and $20 \%$ reported dyspepsia within 14 days prior to baseline. $12 \%$, $10 \%$, and $14 \%$ had stopped an arthritis medication at some time due to abdominal pain. $28 \%$ of the LA patients had changed their arthritis medication in the previous year; compared to $50 \%$ in EUR and $46 \%$ in NA.

Conclusion Because of its size, the VIGOUR cohort is useful for studying regional differences in patient characteristics and treatment strategies. While most measured patient characteristics are similar, differences in the proportions of seropositive patients and patients with erosions and nodules may reflect other differences in the medical characteristics of the populations. This cohort also highlights differences in treatment strategies across 
regions as hydroxychloroquine was more frequently used in LA and injectable gold and sulfasalazine were more frequently used in EUR. The new DMARDs were being used more in the US, probably due to earlier regulatory agency approval.

\section{SAT0256 ECONOMIC IMPACT OF INFLIXIMAB TREATMENT IN RHEUMATOID ARTHRITIS; CORRELATION WITH DISEASE ACTIVITY SCORE}

${ }^{1} \mathrm{~A}$ Kavanaugh, ${ }^{2} \mathrm{~K}$ Patel, ${ }^{2} \mathrm{M}$ Bala, ${ }^{3} \mathrm{~L}$ Noe, ${ }^{4} \mathrm{C}$ Antoni. ${ }^{1}$ Rheumatology, University of California at San Diego, La Jolla, USA; ${ }^{2}$ Biostatistics, Centocor, Inc., Malvern; ${ }^{3}$ Ovation Research Group, Highland Park, USA; ${ }^{4}$ Medicine, University Erlangen-Nuremberg, Erlangen, Germany

\subsection{6/annrheumdis-2001.892}

Background In the ATTRACT trial, rheumatoid arthritis (RA) patients treated with the anti-TNF monoclonal antibody infliximab showed significant improvement in the signs and symptoms of disease (e.g. as measured with using the Disease Activity Score [DAS]); treatment also achieved improvement in quality of life measures and inhibited the progression of structural damage at 54 weeks. The ultimate clinical utility of biologic agents may be impacted by their high acquisition costs. However, RA is itself associated with substantial direct and indirect costs that vary with the severity of disease. Therefore, highly effective therapies for RA may be cost effective.

Objectives To assess the total costs of treating RA, exclusive of the costs of study medication, for patients enrolled in the ATTRACT trial, and to correlate total costs with disease activity and response to therapy using the DAS.

Methods Economic evaluation was performed on patients in the ATTRACT trial over 54 weeks of study. The economic impact of improvement in disease activity was assessed by analysing costs according to clinical response using the DAS. DAS28 was calculated as follows:

$0.56 * \mathrm{SQRT}(\mathrm{TJC})+0.28 * \mathrm{SQRT}(\mathrm{SJC})+0.7 \ln$ (ESR) + $0.014 *$ (pt global (VAS)).

Clinical responses in the trial were characterised as 'None', 'Moderate' or 'Good' using DAS scores at endpoint and change in DAS during the study. Categorical variables were tested using Cochran-Mantel-Haenszel test.

Results Clinical efficacy at 54 weeks was significantly greater among patients receiving infliximab compared to those receiving placebo $(\mathrm{p}=0.001)$. Total cost among all patients with ?Good? DAS response was $\$ 3,836$ compared to $\$ 5,613$ and $\$ 13,125$ among patients with ?Moderate? and ?None? response respectively. Thus, patients who had a ?Good? DAS response cost $\$ 9,289$ less on average, compared to ?None? DAS response. DAS28 response rates and total cost by DAS response are presented below.

\begin{tabular}{llll}
\multicolumn{4}{l}{ Abstract SAT0256 Table 1 } \\
\hline & Good & Moderate & None \\
\hline Pts. evaluated & 110 & 112 & 205 \\
Placebo DAS28 Response & $5.7 \%$ & $15.9 \%$ & $78.4 \%$ \\
Infliximab DAS28 Response & $30.9 \%$ & $28.9 \%$ & $40.2 \%$ \\
Mean \pm SD & $\$ 3,836 \pm \$ 2,893$ & $\$ 5,613 \pm \$ 6,640$ & $\$ 13,125 \pm \$ 29,119$ \\
Median & $\$ 2,965$ & $\$ 3,386$ & $\$ 5,530$ \\
Interquartile range & $(\$ 1,960, \$ 4,856)$ & $(\$ 2,170, \$ 6,130)$ & $(\$ 2,889, \$ 10,501)$ \\
Range & $(\$ 433, \$ 16,717)$ & $(\$ 141, \$ 39,610)$ & $(\$ 226, \$ 350,138)$ \\
\hline
\end{tabular}

Conclusion Improvement in DAS significantly reduced the cost of treating RA patients. In patients with severe RA, therapy with infliximab was effective, as assessed by DAS. Moreover, patients achieving moderate and good DAS responses incurred significantly less costs over 54 weeks of therapy than non-responders. This data shows that among patients with severe RA, highly effective therapy, such as infliximab, can reduce costs. Such therapy may be cost effective.

\section{SAT0257 SOME DATA ABOUT THE HOSPITAL CARE AND DISABILITY RATE OF RA IN HUNGARY}

${ }^{1} \mathrm{P}$ Géher, ${ }^{2} \mathrm{G}$ Héjj, ${ }^{3} \mathrm{~L}$ Kincses. ' Chair of Rheumatology and Physiotherapy, Semmelweis University; ${ }^{2}$ Department of Rheumatology, National Institute of Rheumatology and Physiotherapy, Budapest; ${ }^{3}$ Direction, Center for Healthcare Information of the Ministry of Health, Szekszárd, Hungary

\subsection{6/annrheumdis-2001.893}

Background Based upon population studies the prevalence of RA in Hungary is $1.01 \%$ among the adult population. (The estimated number of RA cases is 84,455 .) RA is characterised by early disability and frequent hospitalisation. Health related quality of life of RA patients can be improved significantly with joint surgery.

Objectives To describe the annual number of hospitalisation due to RA, the annual number of large joint surgery performed among RA patients, the number of new case of RA with permanent disability.

Methods From the national hospital database all cases with the main diagnosis of RA (ICD code group M05 and M06) were selected for analysis. Multiple hospitalisation of the same patients were excluded. The number of disability pension for RA was drawn from the annual report of the National Institute for Medical Experts.

Results There were 2,556,338 hospitalisation in Hungary in 1999. In 5,243 cases (0.2\%) RA was the main diagnosis. This represents the $6.2 \%$ of all RA patients, calculated on the epidemiological data. In this year 7,106 total hip replacement and 2,539 knee replacement were performed, among them there were 75 cases of hip, and 78 cases of knee surgery in RA. Reconstructive surgery represents $2,9 \%$ of the total hospitalisation with RA. There were 3,667 new disability claimants with locomotor diseases (with any of the "M" ICD code) which is $10.6 \%$ of the total new pensionist. RA was the leading cause of disability in 231 (6.2\%) cases.

Conclusion Our hospitalisation data with RA are conflicting with the estimated total number of RA patients. Surgery (as measured by the number of large joint reconstuctive surgery) represents only a small proportion as an indication for hospitalisation with RA. 